\title{
El discurso colonialista en la internacionalización y sostenibilidad: una mirada desde América Latina
}

The colonialist discourse on internationalization and sustainability: A look from Latin America 
El presente artículo busca mostrar los procesos de internacionalización y la implementación de prácticas de sostenibilidad como manifestaciones de colonialismo aplicadas en el contexto latinoamericano que no se ajustan a sus realidades locales. Se resalta la necesidad de abordar estos conceptos desde una mirada propia, local y que tenga en cuenta las singularidades de los problemas de la organización desde la realidad latinoamericana. Así, la internacionalización puede ser concebida de manera que promueva un desarrollo sostenible regional de los llamados países del sur y disminuya la relación de dependencia respecto de la hegemonía del norte, para lo cual se hace necesaria la creación de teorias que tengan en cuenta las particularidades de las empresas de la periferia y resalten sus diferencias con las pertenecientes a las grandes y tradicionales potencias mundiales.

Palabras clave: internacionalización, sostenibilidad, discurso colonialista, colonialidad, colonialismo

This article seeks to show the internationalization process and the implementation of sustainability practices as manifestations of colonialism in the Latin American context which are not adjusted to their local realities. The need to address these concepts from a local perspective that takes into account the uniqueness of the organization's problems from the Latin American reality is highlighted. Thus, internationalization can be conceived in a way that promotes sustainable regional development of the so-called southern countries and reduces the dependency relationship with the northern hegemony, for which it is necessary to create theories that take into account the particularities of the periphery companies and highlight their differences with those belonging to the great and traditional world powers. 


\section{Introducción}

La colonialidad es un fenómeno poco nombrado e incluso se han ignorado sus efectos en los países "en vías de desarrollo» de América Latina. A través de esta se incorporaron como propios pensamientos y saberes eurocéntricos que son utilizados como herramientas para despojar de su propia identidad a las sociedades dominadas (Correa \& Saldarriaga, 2014; Grosfoguel, 2000; Lander, 2000).

En este sentido, el eurocentrismo, impuesto hegemónicamente como perspectiva de conocimiento superior, se presenta como la única alternativa de conocimiento que explica la realidad de estas sociedades latinoamericanas, heredada desde la época de la Conquista; y configura las prácticas sociales, políticas y económicas actuales en América Latina (Amin \& Moore, 1989; Morales et al., 2019; Quijano, 2000a).

A partir de allí, las corporaciones han adoptado prácticas que no les son propias y que buscan delimitar su actuar de la misma forma en que lo hacen las corporaciones de los países "desarrollados». Tal es el caso de las estrategias y modos de internacionalización de las empresas de países emergentes o multilatinas, que se definen como "las empresas multinacionales de América Latina» (Cuervo-Cazurra, 2010, p. 16), cuya internacionalización ha sido analizada a partir de teorías desarrolladas en contextos ajenos a sus realidades (Aguilera et al., 2017). Esta situación empieza a ser vista como poco aplicable y poco útil con la aparición de autores como Mathews (2002), quien argumenta que la internacionalización de las empresas multinacionales de mercados emergentes adaptada a su contexto solo puede ser entendida con la creación de nuevos modelos teóricos.

Por otra parte, discursos como los del desarrollo sostenible y las prácticas de sostenibilidad son también heredados de las llamadas "potencias mundiales», tal como ocurre con la elaboración y aplicación de los estándares de la Global Reporting Initiative (GRI), originarios de Holanda, que son presentados como los estándares de sostenibilidad más adoptados a nivel global para la presentación de prácticas de sostenibilidad en las corporaciones. Es más, «el 93\% de las 250 corporaciones más grandes del mundo informan sobre su desempeño en materia de sostenibilidad" (GRI, 2019).

Debe cuestionarse entonces si temas como la internacionalización y las prácticas de sostenibilidad de estas empresas de países de economías y mercados emergentes, y más específicamente latinoamericanas, pueden ser analizados de una manera lineal dictada desde el centro hacia la periferia; o si, por el contrario, existe la posibilidad de plantear alternativas distintas con consideraciones más complejas que se ajusten a sus propias realidades y que permitan la liberación del «espejo eurocéntrico donde nuestra imagen es siempre, necesariamente, distorsionada» (Quijano, 2000a, p. 242).

De esta manera, el presente artículo busca mostrar que los modos de expansión, el afán de consolidar procesos de internacionalización y la implementación de prácticas de sostenibilidad empresarial son manifestaciones de colonialidad aplicadas en el contexto latinoamericano que no se ajustan a sus realidades, necesidades y aspiraciones locales.

Para iniciar, se abordará el desarrollo conceptual que incluye asuntos de colonialidad del poder y los relaciona con la internacionalización, medición y estandarización de la sostenibilidad, vistas como mecanismos de dominación por parte de los países desarrollados sobre los subdesarrollados. Seguidamente, se presenta una discusión en la 
que se abordan asuntos de la decolonialidad en las instituciones con el propósito de hacer un llamado a establecer estrategias de una manera descentralizada. Por último, se rescatarán algunas conclusiones que resaltan la necesidad de abordar estas concepciones desde una mirada propia, local y singular que permita pensar los problemas de la organización desde la realidad latinoamericana.

\section{Desarrollo conceptual}

\subsection{Colonialidad}

La aparición del sujeto universal bajo la figura eurocéntrica de hombre blanco, europeo y propietario tiene su fundamento en la época de la Conquista, en la que todo pensar y conocimiento que no proviniera de hombres con estas características era considerado inválido (Bell, 1988; Helms, 1997). Por lo anterior, el racismo aparece como un fenómeno excluyente no solo por color de piel, sino por el propio ser y saber (Correa \& Saldarriaga, 2014).

De allí que la colonialidad se haya expandido a todos los ámbitos de lo humano de forma que, por un proceso de transferencia, los dominantes determinan el actuar de los dominados por medio del traspaso de saberes y prácticas de Europa y Estados Unidos a otras regiones del mundo (Smith, 2012). Se evidencia entonces la supremacía del relato generado desde el punto de vista de los dominantes, con base en una manera de pensar que opaca y discrimina los saberes ancestrales de los pueblos latinos (Leonardo, 2004; Wills, 2013).

Como lo indica Quijano, la colonialidad

Es uno de los elementos constitutivos y específicos del patrón mundial de poder capitalista. Se funda en la imposición de una clasificación racial/étnica de la población del mundo como piedra angular de dicho patrón de poder y opera en cada uno de los planos, ámbitos y dimensiones, materiales y subjetivas, de la existencia social cotidiana y a escala societal. Se origina y mundializa a partir de América (2000a, p. 342).

En este sentido, el eurocentrismo propio de este discurso colonial refleja los intereses capitalistas del mundo moderno y su modo de producir conocimiento, que sobrepasa el mundo premoderno, precapitalista o no europeo, catalogado mediante etnias y tribus, a diferencia de los Estado-nación desarrollados en Europa (Taylor, 1992). De allí que la clasificación social de las personas se realice bajo las categorías de género, raza y trabajo (Ammot \& Matthaei, 1996), y en torno de dos ejes centrales: el control de la producción de recursos de sobrevivencia social, que contempla aspectos como fuerzas, recursos naturales, y recursos y productos del trabajo; y el control de la reproducción biológica de la especie, que implica el control del sexo (Quijano, 2000b).

Esta clasificación social, que permite naturalizar las relaciones de poder y, por ende, su colonialidad, invadió todas las áreas de la existencia social, dando origen al hegemonismo y la estratificación de sociedades en superiores e inferiores, estas últimas consideradas como "periferia», dependientes de una manera "histórico-estructural» de sus colonizadores, quienes son denominados como "el centro», y lideran y acumulan el beneficio a escala global (Lenski, 2013). 
Es de esta manera, como argumenta Quijano, que las sociedades colonizadas son despojadas de "sus saberes intelectuales y de sus medios de expresión exteriorizantes u objetivantes. Fueron reducidos a la condición de gentes rurales e iletradas» (2000b, p. 378). Este despojo de saberes se realiza a partir de un proceso de transculturación, que puede ser descrito como un "conflicto social entre lenguajes y cosmologías en posiciones hegemónicas y subalternas respectivamente» (Mignolo \& Schiwy, 2003, p. 21), el cual fue impuesto en los pueblos latinos.

La colonialidad, además, deshumaniza al hombre civilizado, ya que el proceso de colonialidad a nivel personal o empresarial se fundamenta en el desprecio por el otro, en su no reconocimiento y en resaltar su diferencia, haciendo realmente diferente a quien lo aplica. Esta invita, también, a imponer relaciones de dominación y sumisión que generan un sentimiento de inferioridad sobre el oprimido (Lester, 2012).

La batalla decolonial debe establecer un nuevo orden con diferentes formas de vincular el mundo de una manera disruptiva y revolucionaria, generando nuevas identidades; un orden en el que cada forma de organización tenga un acceso equitativo a los recursos para evitar desigualdades entre unos y otros (Tuck \& Yang, 2012). En particular, América Latina y sus relaciones dentro del mundo se entienden como un espacio con relaciones donde predomina la colonialidad del poder, lo que explica su realidad bajo la mirada europea, desajustada de su propio contexto. "Debido a ello, los problemas no han podido ser nunca ceñidamente planteados y mucho menos adecuadamente resueltos» (Quijano, 2014, p. 207). En este sentido, se hace necesario que América Latina establezca su identidad alejada del eurocentrismo que no le es propio y que busque la democratización de los recursos de producción; es decir, una redistribución de las relaciones de poder en el Estado que conlleven a su desarrollo continuado (Coronil, 2015).

El «bien vivir» es definido por Quijano como un "complejo de prácticas sociales orientadas a la producción y a la reproducción democráticas de una sociedad democrática, un otro modo de existencia social, con su propio y específico horizonte histórico de sentido, radicalmente alternativos a la Colonialidad» (2014, p. 847) propuesto por «indígenas latinoamericanos». Este modelo se presenta como una alternativa de vida social que responde a la crisis actual del nuevo periodo histórico, caracterizado por problemas de desigualdades desproporcionadas y exclusión, así como de explotación social y de la naturaleza, que ponen en riesgo la reproducción de las condiciones de vida y deben ser superados por medio de la búsqueda de la defensa de la naturaleza y la vida humana frente a toda forma de dominación.

Adicionalmente, se requiere una transformación en el proceso de producción de conocimiento, que debe abarcar desde sus metodologías de investigación hasta la aplicación de este en la organización para lograr una decolonialidad en el conocimiento y en todos los ámbitos de las sociedades. De esta manera, se puede lograr un desarrollo ético e inclusivo enmarcado en un contexto externo al eurocentrista. Para alcanzarlo, es posible acudir a las recomendaciones desarrolladas por Girei (2017), quien argumenta que existen seis temas claves que pueden ser útiles al momento de generar conocimiento con características decoloniales. El primero es el enfoque cualitativo, que incluye también posiciones interpretativas e inductivas que permiten un mayor compromiso con diversos participantes y grupos de análisis. El segundo es la orientación abierta, en respuesta 
a la investigación empírica y su marco teórico, útil para ampliar las perspectivas y aprender de todas las partes involucradas sin restringir su variedad. El tercer tema es la reflexividad, la misma que invita a reconocer que el conocimiento no es neutro y que no puede ser separado de las circunstancias en las que se produce, donde los intereses y las acciones de los investigadores tienen un impacto en su generación. En cuarto lugar, se menciona el reconocimiento de la blancura y otras asimetrías, que conlleva a una inclusión de otras realidades, contextos, historias y posturas de una manera más amplia, evitando su eliminación en la investigación, como usualmente se hace. El quinto tema es la contextualidad radical, que invita a prestar atención simultánea a las dimensiones históricas del contexto en el que se lleva a cabo la investigación para contrarrestar los enfoques descontextualizados, que tienen efectos perjudiciales en las organizaciones no anglosajonas. Y, finalmente, se encuentra el tema micro/meso/macro, lo que involucra una investigación que se mueve desde lo maso, pasa por lo meso y llega hasta lo micro $-\mathrm{y}$ viceversa- para evitar el silenciamiento de perspectivas alternativas y el sostenimiento de desigualdades sociales.

De esta manera, entonces, se podrá alcanzar una decolonialidad de conocimiento, que permita generar nuevas propuestas, miradas y enfoques en los procesos de internacionalización de empresas multilatinas, desarrolladas a partir de sus propias características y contextos; así como la aplicación de prácticas que busquen la protección de los recursos y las especies propias de esta región, alineadas a sus ambientes naturales e institucionales, y considerando sus realidades históricas.

\subsection{Internacionalización de empresas de países de economías y mercados emergentes}

Las asimetrías de poder y las desigualdades históricas entre los contextos angloamericanos y europeos frente el resto del mundo se evidencian también en la manera de investigar, que adopta teorías y metodologías eurocentristas. La investigación en estudios organizacionales se ha originado principalmente en Estados Unidos e Inglaterra, y esto incluye también el desarrollo de teorías y enfoques críticos (Acedo et al., 2006; Weick, 1996). Debido a esta concepción, las voces de los investigadores, organizaciones y sociedades del resto del mundo se han visto opacadas, poco escuchadas e incluso ignoradas, tal como ocurre con América Latina. Se sufre entonces de una descontextualización y un ahistoricismo que separa a las sociedades de sus contextos y las incluye en una fábrica cultural y social más grande.

A pesar de esto, han surgido nuevos enfoques que estudian las organizaciones con una mirada diferente, como las diversas propuestas de internacionalización de empresas de mercados emergentes, que se diferencian de los modelos y procesos que reflejan la lógica de los países tradicionalmente industrializados y buscan su inclusión en la cúpula del conocimiento.

Tal como ocurre con el concepto de distancia psicológica, en la que los individuos prestan mayor importancia a lo que se percibe más cerca en términos espaciales y temporales (Tatsuo \& Aguilera, 2017), las organizaciones también consideran el concepto de distancia para tomar decisiones de internacionalización y selección de mercados (Lezana et al., 2020). En este sentido, la distancia cultural, geográfica y administrativa entre país de origen y país de destino cobra relevancia cuando se analizan las elecciones de las compañías 
para desarrollar sus actividades en mercados percibidos como "menos distantes» $y$, al mismo tiempo, puede determinar las dinámicas propias de las interrelaciones entre los llamados países del norte y del sur.

No obstante, la internacionalización, entendida como un proceso de mayor participación de las empresas en los mercados internacionales - lo que conlleva la necesidad de adaptar los productos o servicios de las compañías a los gustos y preferencias de los consumidores en el exterior, al igual que sus prácticas organizacionales-, puede ser considerada como un mecanismo de colonialidad. Frente a ello, existe la posibilidad de concebir una internacionalización diferente que promueva el desarrollo regional de los países del sur y disminuya la relación de dependencia con el norte, para lo cual se hace necesaria la creación de teorías que demuestren las particularidades de las empresas de la periferia y resalten sus diferencias respecto a las pertenecientes a las grandes potencias (Moreno \& Espinosa, 2018).

En este sentido, el concepto de empresas multilatinas emerge como contraposición a la tradicional versión de las empresas multinacionales pertenecientes a países desarrollados. En esta línea, Cuervo-Cazurra argumenta que las empresas multilatinas no son un fenómeno reciente, pero sí lo es el interés por estas. A pesar de su antigüedad, aún existen brechas de conocimiento sobre los procesos de transformación de estas empresas, tal vez por la poca relevancia que los investigadores anglosajones les han otorgado. En esa línea, el autor señala que, «En general, la literatura de negocios internacionales ha prestado poca atención a Latinoamérica y sus empresas multinacionales» (2010, p. 25).

Esto se logra observar con el estudio de las diferentes teorías de internacionalización propuestas por diversos autores que, curiosamente, son originarios del mundo anglosajón. Siguiendo este razonamiento, Zhang et al. (2014) clasifican las teorías de internacionalización en cuatro categorías diferentes: internalización y costo de transacción, teoría basada en recursos, organización industrial y teoría de incentivos administrativos.

En la tabla 1 se puede observar la clasificación de las diferentes teorías de internacionalización que han generado mayor impacto teórico y práctico, incluyendo su respectivo autor, año de creación y país de procedencia.

Tabla 1. Teorías de internacionalización según sus categorías, autores, año y país

\begin{tabular}{l|l|l|l} 
Categoría & Autor & Año & País \\
\hline Ciclo de vida del producto & Vernon & 1966 & Estados Unidos \\
\hline Internalización y costos de transacción & Buckley y Casson & 1976 & Inglaterra \\
\hline Teoría del control & Hymer & 1976 & Canadá \\
\hline Internacionalización gradual-secuencial & Johanson y Vahlne & 1977 & Suecia \\
\hline $\begin{array}{l}\text { OLI paradigm (propiedad, localización e interna- } \\
\text { cionalización) }\end{array}$ & Dunning & 1977 & Inglaterra \\
\hline Modelo de conocimientos y recursos & Kogurt y Zander & 1993 & Estados Unidos y Suecia \\
\hline Nacidas globales & Oviatt y McDougall & 1994 & Estados Unidos \\
\hline LLL (vinculación, apalancamiento y aprendizaje) & Mathews & 2006 & Australia
\end{tabular}


Como se puede observar, las teorías de internacionalización que han sido aplicadas en las empresas de los países del mundo, incluyendo las del sur, tienen un origen anglosajón, lo cual es muestra de una colonialidad que se refleja en su estudio y en el conocimiento que se ha generado sobre las mismas a partir de realidades no propias. Tal como lo argumentan Aguilera et al. (2017), América Latina es una región poco investigada que tiene el potencial de ofrecer nuevas e importantes perspectivas sobre la internacionalización de empresas de mercados emergentes, particularmente en comparación con la experiencia de empresas de otras regiones. De esta manera, se hace necesario descubrir los aspectos que son aplicables a estas empresas a partir de la teoría existente y también cuáles no, por lo que se necesita el desarrollo de nueva teoría (Ramamurti, 2012). Es así como se espera que se pase del mero criticismo y reconocimiento de diferencias entre unas teorías y otras a una aceptación, validación y legitimización del conocimiento desarrollado en la periferia.

Sin embargo, según Girei (2017), se debe reconocer que la tradición eurocentrista, constantemente reproducida en los países del sur, se perpetúa también por el rol de estos como receptores de ayuda y soporte por parte de organismos e instituciones internacionales. Dicho rol ejerce presión respecto a la adopción de teorías y prácticas desarrolladas en contextos ajenos, como es el caso de las prácticas de sostenibilidad en los países sudamericanos y las estrategias de internacionalización que estos deben adoptar en su intento por tener un nivel de participación en los competitivos mercados del Norte. Debido a ello, se debe abogar por una ética de la liberación que enmarque los fines, medios y métodos de la acción estratégica de las organizaciones para lograr su mayor fin: la emancipación del ser (Dussel, 1998).

\subsection{Desarrollo sostenible}

La interpretación de desarrollo también ha sido considerada como una práctica colonial, ya que en esta se asume una postura de progreso concebida a partir del mundo occidental. Casos de regiones como África y América Latina demuestran la influencia de las ideas de crecimiento económico y capitalista como sinónimos y pruebas de un desarrollo de las sociedades que no se adapta, a la par que tampoco explica su propio crecimiento y desarrollo. Como lo plantea Godelnik (2021), existe la necesidad de cambiar a un modelo de sostenibilidad que no solamente vele por el bienestar de los accionistas, sino que busque satisfacer a los diferentes grupos de interés. Este cambio de paradigma involucrará un empoderamiento y una liberación para dar paso a las nuevas identidades de los pueblos, las organizaciones y los individuos.

A lo largo del tiempo, el discurso de desarrollo y sostenibilidad ha sido dominado y liderado por prácticas y teorías creadas en países anglosajones que proponen soluciones basadas en sus propios contextos, pero que son aplicadas en el resto del mundo (Foo, 2007). Esta situación lleva a la búsqueda de diferentes maneras de conocimiento, que se justifican como factores clave dada la creciente preocupación internacional con respecto a los modelos neoliberales para el desarrollo, los cuales incluyen las reformas que dan prioridad al crecimiento económico del sector privado y disminuyen las garantías sociales a cargo del Estado (Bowers Du Toit, 2018). 
Frente a esta realidad, se hace necesario explorar alternativas diferentes que integren una creencia en la identidad indígena autónoma, en contraposición a su definición en relación con las ideologías y culturas occidentales. A la par, se hace patente la importancia de un énfasis en la construcción de economías a nivel local y comunitario, de promover economías distantes a las ideologías capitalistas y de aquellas formas colectivas de gobierno que incrementen el apoyo a los movimientos sociales para que, de esta manera, el desarrollo se entienda y aplique a partir del contexto propio.

El discurso del desarrollo no fue construido bajo el marco y el lenguaje de la emancipación y la liberación, sino con un vocabulario de "caridad, experticia técnica, neutralidad y paternalismo" (Bowers Du Toit, 2018, p. 32) enmascarado en una agenda participativa, como es el caso de los Objetivos de Desarrollo Sostenible (ODS) surgidos a raíz del concepto de desarrollo sostenible. Cabe precisar que este último es definido como la satisfacción de «las necesidades de la generación presente sin comprometer la capacidad de las generaciones futuras para satisfacer sus propias necesidades" (Naciones Unidas, 1987). Se evidencia entonces que, a lo largo de los años, se han forjado diferentes concepciones de desarrollo que abarcan diversas propuestas de aplicación organizacional (ver tabla 2).

Tabla 2. Visiones sobre el concepto de desarrollo sostenible

\begin{tabular}{l|l|l} 
Propuesta & Autor & Año \\
\hline $\begin{array}{l}\text { Satisfacción de las necesidades de la generación presente sin com- } \\
\text { prometer la capacidad de las generaciones futuras para satisfacer } \\
\text { sus propias necesidades }\end{array}$ & Naciones Unidas & 1987 \\
\hline Triple bottom line o «triple cuenta de resultados» & John Elkington & 1994 \\
\hline $\begin{array}{l}\text { Vocabulario de caridad, experticia técnica, neutralidad y paternalismo } \\
\text { Con base en tres dimensiones que buscan equilibrar lo económico, } \\
\text { social y ambiental }\end{array}$ & Gowers Du Toit & 2018 \\
\hline
\end{tabular}

Fuente: elaboración propia.

La idea de desarrollo sostenible se basa en tres dimensiones que buscan equilibrar lo económico, social y ambiental (Gomez-Trujillo \& Gonzalez-Perez, 2020); sin embargo, al indagar sobre esta propuesta, se encuentra que la misma proviene del concepto de triple bottom line o «triple cuenta de resultados», que fue presentado en 1994 por John Elkington, un autor y asesor inglés, líder en temas de responsabilidad social corporativa y desarrollo sostenible, con una gran experiencia en emprendimiento y consultoría empresarial y política. Se resalta, además, su participación en diferentes juntas y su experiencia investigativa, lo cual justifica la acogida de su teoría tanto en su país de origen como en economías subdesarrolladas y en la región latinoamericana.

La triple cuenta de resultados es la fundamentación para la creación de propuestas de desarrollo sostenible y la responsabilidad social empresarial. A partir de su difusión, tanto en países anglosajones como en el resto del mundo, se han desarrollado iniciativas que intentan reforzar sus presunciones, como los ya mencionados ODS, que se presentan como un 
Llamado universal a la adopción de medidas para poner fin a la pobreza, proteger el planeta y garantizar que todas las personas gocen de paz y prosperidad [...] Los ODS son una agenda inclusiva. Abordan las causas fundamentales de la pobreza y nos unen para lograr un cambio positivo en beneficio de las personas y el planeta (Naciones Unidas, 2015).

Adicionalmente a esto, se han creado estándares para la presentación de reportes que den cuenta del compromiso con el desarrollo sostenible por parte de las empresas. Tal es el caso de la GRI, una organización internacional no gubernamental (ONG) creada en Boston en 1997 que tiene como objetivo impulsar los reportes de sostenibilidad como herramienta para la planificación, medición, evaluación y comunicación de los avances e impactos en aspectos sociales, económicos y ambientales de una organización. La GRI produce la estructura de reportes de sostenibilidad más utilizada en el mundo, incluyendo a las empresas multilatinas, las cuales, por su herencia colonial, miden su compromiso con base a estos estándares, que no dan fe de su concepción de desarrollo ni de sus realidades locales.

Los anteriores ejemplos revelan la pretensión de universalización de la idea de desarrollo, que involucra tanto a actores públicos como privados para caminar en la misma dirección, lo cual refuerza la colonialidad del conocimiento y del poder en todos los rincones del mundo. Surge entonces la necesidad de que las empresas multilatinas emprendan estrategias que generen sus propios objetivos de desarrollo sostenible, alineados — por ejemplo- a la preservación las tradiciones y culturas propias de estos pueblos. Estos nuevos enfoques y prácticas podrían dar respuesta a sus problemas y, como lo manifiesta Ibarra (2006), permitirán pensar en el problema de la organización desde el centro y no desde las orillas.

Es deber, entonces, de las organizaciones decidir si sus políticas y prácticas continuarán inmersas en la dualidad superior-inferior, o si, por el contrario, se generará un compromiso real con el conocimiento y los sistemas locales que les permita responder a sus propios retos y a la manera en que entienden sus contextos socioculturales. La agenda debe presentarse así no como un ejercicio de caridad o participación conjunta, sino como un mecanismo de liberación y restauración total, pasando del desarrollo basado en proyectos al entrenamiento y equipación de las organizaciones en búsqueda de su defensa.

Es hora, pues, de revelar el lado oculto de las propuestas de desarrollo y reducción de la pobreza, que encuentran soluciones marginales y desarticuladas con una búsqueda veraz de la emancipación de los pueblos oprimidos por sus sistemas sociales, políticos y económicos. En otras palabras, es tiempo de «dejar de ser lo que no somos» (Quijano, 2000c, p. 242).

\section{Discusión}

Como se pudo evidenciar, la colonialidad se revela al interior de las instituciones de los pueblos latinos, que abarcan organizaciones colonizadas con gente colonizada (García, 2018). Se hace entonces necesario reconocer la historia de colonialismo para moverse $o$ transformarse en modelos organizativos fundamentados en la decolonialidad. Esta autora propone un modelo organizativo para la decolonialidad que contiene nueve elementos que 
abarcan aspectos internos y externos de las instituciones: propósito, misión, afiliación, tecnología, gobernanza, normas de la comunidad, justicia y responsabilidad, estructura de incentivos y, finalmente, gestión de límites externos.

Cada uno de estos elementos contiene un significado y concepción a partir de la decolonialidad. El propósito es planteado como una manera de incluir el entendimiento racial y cultural propio y de los otros como un fin de la organización que busca aumentar el conocimiento de los valores, ideologías y epistemologías de los pueblos de América. La misión, por su parte, debe contener elementos antirracistas y antiopresivos para empoderar a las comunidades nativas $u$ oprimidas. En tercer lugar, la afiliación se fundamenta en una ideología de mezcla cultural y racial que respeta las diferencias entre sus miembros; mientras que la tecnología revela la necesidad de desarrollar herramientas propias que se ajusten al contexto local, fundadas en el principio de liberación. El quinto elemento, la gobernanza, abarca aspectos como reglas, autoridad y estructura organizacional, que deben ser ajustados a los estándares de la comunidad bajo principios de integridad y pluralismo. Por otro lado, las normas de la comunidad deben ser desarrolladas bajo un criterio de protección y progreso comunitario, incluyendo a todos sus miembros; en tanto que la justicia y responsabilidad se basan en la construcción de redes y relaciones para la restauración de la comunidad, reconociendo a todos los miembros como igualmente aptos para desarrollar valores y resolver problemas en los procesos de justicia. El siguiente elemento, la estructura de incentivos, se debe alinear a la misión y al propósito de la organización con recompensas para aquellos que actúan según estos lineamientos, dejando de lado el individualismo y buscando mantener grupos diversos al interior de la organización. Finalmente, la gestión de límites externos debe realizarse incluyendo a todos los grupos de interés internos y externos, y está fundamentada en el colectivismo para emprender iniciativas de mutuo beneficio (García, 2018). Este último elemento es sumamente importante, ya que —como lo mencionan Gonzalez-Perez et al. (2021)_, en momentos de crisis, la gestión de los grupos de interés se basa en la responsabilidad social, lo que podría resultar en mejoras en las relaciones con estos y en la definición de los límites organizacionales.

Este marco de aplicación para la decolonialidad en las instituciones tiene implicaciones a nivel interno y externo, ya que representa un llamado a reconocer la colonialidad del poder para direccionar el propósito y las estrategias de la institución de una manera descentralizada que involucre diversos actores y permita el desarrollo de la democracia interna. Para lograr su liberación y un desarrollo epistemológico propio es necesario, además, iniciar por un reconocimiento de su estado como instituciones colonizadas; es decir, organizaciones de las cuales se espera un comportamiento similar al de las instituciones de los países desarrollados.

A partir de aquí se pueden generar propuestas alternas para las prácticas y reportes de sostenibilidad de las empresas multilatinas y sus estrategias de internacionalización, buscando que estén alineadas a los modelos organizativos de características decoloniales y a sus propias características y percepciones de distancias psicológicas. Estas, a su vez, deben tener por objeto conseguir el fortalecimiento regional por medio acuerdos sur-sur para generar un desarrollo y crecimiento equitativo que pueda igualar o superar el disfrutado por las empresas de países desarrollados, ya que, según Gonzáles-Miranda et al. (2017), 
interpretar los problemas de la realidad local es indispensable para considerar la realidad latinoamericana.

Tal es el caso del Centro de los Objetivos de Desarrollo Sostenible para América Latina (CODS), que se presenta como una propuesta para generar una voz propia latinoamericana y un sitio de encuentro y pensamiento sobre los ODS en conjunto con diferentes actores públicos y privados de América Latina y el Caribe (CODS, s.f.).

Lo mismo sucede con Interconexión Eléctrica S.A. -también conocida como Grupo ISA-, multilatina colombiana que opera en la industria de las telecomunicaciones, energía y vías. Esta empresa ha vivido un proceso de transformación y apropiación en temas de internacionalización y sostenibilidad que la han convertido en líder y referente mundial en el desarrollo de estrategias (ISA, 2020). Este grupo multilatino ha superado las dificultades institucionales de su país de origen para transformarlos en ventaja competitiva en mercados externos. Puntualmente, el proceso de internacionalización de la compañía se caracterizó principalmente por adquisiciones en países de la región con una cercanía geográfica, fortaleciendo el crecimiento y la interconexión de países latinoamericanos. Por otra parte, su estrategia de sostenibilidad se ha caracterizado por contener un enfoque propio denominado "Nuestro futuro inspirado por el valor sostenible» (ISA, 2019). Allí, la empresa se inspira por el acrónimo VIDA (verde, innovación, desarrollo y articulación) para el desarrollo sostenible. Lo anterior demuestra la capacidad de las empresas multilatinas para generar estrategias propias, disruptivas y que generan valor tanto para la organización como para la región; y que influyen, además, en su desempeño financiero y reputacional (Gomez-Trujillo et al., 2020).

En este sentido, el rol del Estado es fundamental en el diseño de estrategias que promuevan e impulsen la creación de este tipo de empresas. En el caso colombiano, la violencia y la incertidumbre política han propiciado la internacionalización de sus empresas de una forma única que les permite desarrollar capacidades para superar estas desventajas de su país de origen (Cuervo-Cazurra, 2016). De igual manera, las iniciativas institucionales impulsan el desarrollo sostenible, lo que permite a las empresas adoptar la sostenibilidad como una bandera de conquista en mercados internacionales. Esto podría convertirse en un impulsor para la generación de acuerdos entre países latinoamericanos destinados a afianzar el crecimiento sostenido y coordinado como parte de un intento de generar una voz propia y desarticulada de las impuestas por las grandes potencias. A su vez, iniciativas de este tipo pueden ocasionar efectos positivos, tanto a nivel público como privado, al servir intereses particulares y colectivos de la sociedad.

Es así como la lucha por la emancipación y la decolonialidad conlleva una búsqueda de innovación y desarrollo propio que incluye empresas de diferentes sectores, productoras, comercializadores y manufactureras de bienes y servicios, además de diferentes grupos sociales, como los 522 pueblos indígenas existentes en América Latina.

Uno de estos pueblos es el emberá chamí de la vereda Las Tazas, parte del municipio de Marsella en Risaralda, Colombia, cuyos saberes ancestrales y artesanales mejoran la regeneración de su identidad cultural mediante un ejercicio de recuperación de prácticas tradicionales para propiciar el desarrollo de sus dinámicas culturales y económicas (Loaiza, 2018). De esta manera, se evidencia que es necesario analizar el contexto sociopolítico para comprender las ideas, creencias y paradigmas que influyen en la 
sustentabilidad de los saberes ancestrales y artesanales en las sociedades contemporáneas (Orellana \& Rivera, 2019).

Además, es importante resaltar que este tipo de iniciativas abarcan desde el uso de materias primas hasta el desarrollo de bienes intermedios y que, más allá de la generación de energías limpias y renovables, buscan una sostenibilidad a largo plazo que incluye las aristas sociales y económicas de estos grupos sociales.

\section{Conclusiones}

El presente artículo busca servir como base para los tomadores de decisiones de organizaciones públicas y privadas en el análisis de sus procesos de internacionalización y la implementación de prácticas de sostenibilidad, desde un enfoque propio que considere las características particulares de su contexto y sus capacidades. Asimismo, este trabajo tiene la finalidad de realizar una contribución teórica para las disciplinas de las ciencias de la gestión, específicamente en la disciplina de los negocios internacionales y la sostenibilidad; $y$, en paralelo, hacer un llamado al desarrollo de literatura en torno al management, especialmente en la región de América Latina.

Se concluye entonces que son las epistemologías propias del sur las que abogarán por el reconocimiento de sus pueblos a través de un proceso de decolonialidad del poder que permita su reconocimiento como iguales ante los países desarrollados. De igual manera, se hace necesaria la elaboración de procesos de internacionalización que busquen un crecimiento regional del Sur y, a la par, fortalezcan la aplicación de prácticas de sostenibilidad ajustadas al contexto y a los recursos propios de las empresas y los pueblos latinos.

La lucha contra la dominación implicará la disputa por la destrucción de la colonialidad del poder, relacionada con el poder capitalista al ser este el eje central de explotación, discriminación y acumulación en las relaciones sociales. Ello obliga a pensar en vías alternas de liberación individual y social que otorguen a las personas y a las sociedades el control de lo que les ha sido arrebatado.

Lo anterior se puede entender como el inicio de una "teoría del sur» (Ndhlovu, 2016), la cual invita al desarrollo de perspectivas teóricas a partir de las realidades propias de los países del Sur dentro de un sistema interconectado de conocimiento, política, economía y cultura global. Esta proposición surge como muestra de que existen otros caminos en la construcción de teoría que ejemplifican la inclusión de los pueblos para trascender los efectos de las teorías «occidentales». Cabe precisar que esta teoría no aspira a la suplantación de las ya mencionadas teorías «occidentales» ni a establecerse como el único camino para la generación de conocimiento, sino que representa una manera de repensar las teorías actuales y su relación con el conocimiento, la democracia, el poder y la identidad de todas las comunidades del mundo, lo que involucra la experiencia de toda la población. Esta teoría necesita entonces ser incluida entre las ideas de desarrollo y progreso social, cuestionando el monopolio y la universalización de las tendencias epistemológicas del Norte. En otras palabras,

el pensamiento decolonial no solo se preocupa por la necesidad de nuevas ideas. Más bien, va un paso más allá para generar una forma de pensar completamente nueva: sobre 
los idiomas, sobre las identidades culturales, sobre los regímenes de conocimiento y producción de conocimiento, y sobre todo lo que hacemos (Ndhlovu, 2015p. 36).

Finalmente, se necesita estudiar el lenguaje, lo local y lo singular, «desarrollando investigaciones sobre una multitud de fenómenos, discursos y prácticas que tienen lugar en y entre las organizaciones, y que podrían sentar las bases de una aproximación propiamente latinoamericana al estudio de las organizaciones» (Saavedra-Mayorga et al., 2017, p. 577) desde una mirada práctica e investigativa. Este desarrollo no debe, pues, ser enfocado meramente al estudio de la internacionalización y sostenibilidad de las empresas de países emergentes como los latinoamericanos, sino a todo tipo de organizaciones y teorías que tengan como fin un reconocimiento de identidades que han sido olvidadas y oprimidas para propiciar su resurgimiento como iguales en el mundo actual. 


\section{bibliografía}

Acedo, Francisco J., Barroso, Carmen, Casanueva, Cristóbal, \& Galán, José L.

2006

Co-Authorship in Management and

Organizational Studies: An Empirical and

Network Analysis. Journal of Management

Studies, 43(5), 957-983

Aguilera, Ruth, Ciravegna, Luciano, Cuervo-Cazurra, Alvaro, \& Gonzalez-Perez, Maria A.

2017 Multinationals and the internationalization of Latin American Firms. Journal of World Business, 52(4), 447-460

Amin, Samir, \& Moore, Russell. Eurocentrism. NYU Press.

Amott, Teresa \& Matthaei, Julie.

Race, Gender and Work: A Multi-cultural

Economic History of Women in the United States. Boston: South End Press.

\section{Bell, Derrick.}

White Superiority in America: Its legal legacy, its economic costs. Villanova Law Review, 33(5), 767-779.

\section{Bowers Du Toit, Nadine.}

Decolonising Development? Re-claiming

Biko and a Black Theology of Liberation within the context of Faith Based Organisations in South Africa. Missionalia: Southern African Journal of Missiology, 46(1), 24-35. https://doi.org/10.7832/46-1-219

\section{Centro de los Objetivos de} Desarrollo Sostenible para América Latina (CODS).

s.f. ¿Qué es el CODS? https://cods.uniandes. edu.co/sobre-el-cods/

\section{Coronil, F.}

2015

Latin American Postcolonial Studies and Global Decolonization. En Postcolonial Studies: An Anthology (pp. 175-192). John Wiley \& Sons.

Correa, Mario E., \& Saldarriaga, Dora C.

2014 El epistemicidio indígena latinoamericano. algunas reflexiones desde el pensamiento crítico decolonial. Revista CES Derecho, 5(2), 154-164.

\section{Cuervo-Cazurra, Alvaro.}

2010

Multilatinas. Universia Business Review, (25), 14-33. https://www.redalyc.org/ pdf/433/43312280002.pdf

\section{Cuervo-Cazurra, Alvaro.}

2016

Multilatinas as sources of new research insights: The learning and escape drivers of international expansion. Journal of Business Research, 69(6), 1963-1972.

\section{Dussel, Enrique.}

Ética de la Liberación en la edad de la globalización y la exclusión. Madrid: Trotta.

\section{Elkington, John.}

Towards the Sustainable Corporation:

Win-Win-Win Business Strategies for

Sustainable Development. California

Management Review, 36(2), 90-100.

https://doi.org/10.2307/41165746

\section{Foo, Min.}

Stakeholder engagement in emerging economies: considering the strategic benefits of stakeholder management in a cross- cultural and geopolitical context. Corporate Governance, 7(4), 379-387. https://doi. org/10.1108/14720700710820461 


\section{bibliografía}

García, Gina.

2018 Decolonizing Hispanic-Serving Institutions:

A Framework for Organizing. Journal of

Hispanic Higher Education, 17(2), 132-147.

https://doi.org/10.1177/1538192717734289

\section{Girei, Emanuela.}

Decolonising management knowledge:

A reflexive journey as practitioner and

researcher in Uganda. Management

Learning, 48(4), 453-470.

\section{Global Reporting Initiative (GRI).}

About GRI. https://www.globalreporting.org/ information/about-gri/Pages/default.aspx

\section{Godelnik, Raz.}

Rethinking Corporate Sustainability in the Era of Climate Crisis: A Strategic Desing Approach. Palgrave Macmillan. https://doi. org/10.1007/978-3-030-77318-2

\section{Gomez-Trujillo, Ana Maria, \& Gonzalez-Perez, Maria Alejandra.}

What do we know about organizational sustainability and international business? Management of Environmental Quality: An International Journal, 31(2). https://doi. org/10.1108/MEQ-08-2019-0173

\section{Gomez-Trujillo, Ana Maria, Velez-Ocampo, Juan, \& Gonzalez-Perez, Maria}

\section{Alejandra.}

A literature review on the causality
between sustainability and corporate
reputation: What goes first? Management
of Environmental Quality: An International
Journal. https://doi.org/10.1108/MEQ-09-
2019-0207

Gonzales-Miranda, Diego R., \& Ramírez Martínez, Guillermo.

Los estudios organizacionales en

Latinoamérica. Una realidad fragmentada en busca de una identidad. En S. R. Clegg,

C. Hardy, T. B. Lawrence, W. R. Nord, G

Ramírez Martínez y D. R. Gonzales-Miranda (eds.), Tratado de estudios organizacionales (vol. I, pp. 23-40). Medellín: UAM,

Remineo, Editorial Universidad Eafit, SAGE.

Gonzalez-Perez, M. A., Cordova, M., Hermans, M., Nava-Aguirre, K. M., Monje-Cueto, F., Mingo, S., Tobon, S., Rodriguez, C. A., Salvaj, E. H., \& Floriani, D. E.

Crises conducting stakeholder salience:

shifts in the evolution of private universities' governance in Latin America. Corporate Governance, 21(6). https://doi.org/10.1108/ CG-09-2020-0397

\section{Grosfoguel, Ramón.}

2000

Developmentalism, Modernity, and Dependency Theory in Latin America. Nepantla: Views from South, 1(2), 347-374.

\section{Helms, Janet E.}

Toward a Model of White Racial Identity Development. College Student Development and Academic Life: Psychological, Intellectual, Social and Moral Issues (pp. 207-224). Nueva York: Routledge.

\section{Ibarra Colado, Eduardo.}

¿Estudios Organizacionales en América Latina? Transitando del centro a las orillas. En E. de la Garza Toledo (ed.), Teorías Sociales y Estudios del Trabajo. Nuevos Enfoques (pp. 88-117). Ciudad de México, México: Anthropos, UAM-I. 


\section{bibliografía}

ISA.

2019

Nuestro futuro inspirado por el Valor

Sostenible. https://www.isa.co/es/valor-

sostenible/

ISA.

2020

Nuestra Compañía. https://www.isa.co/es/ grupo-isa/

\section{Lander, Edgardo.}

2000 Eurocentrism and Colonialism in Latin American Social Thought. Nepantla: Views from South, 1(3), 519-532

\section{Lenski, Gerhard E.}

2013

Power and Privilege: A Theory of Social

Stratification. Carolina del Norte: UNC

Press Books.

Leonardo, Zeus.

2004

The Color of Supremacy: Beyond the discourse of white privilege. Educational Philosophy and Theory, 36(2), 137-152.

\section{Lester, Alan.}

2011

Humanism, race and the colonial frontier.

Transactions of the Institute of British

Geographers, 37, 132-148.

\section{Lezana, Bruce, Cancino,} Christian, Guede, Belén, \& Salazar-Elena, Juan C.

Psychic Distances and the Export Success

of Born Global Firms. The Mexican Journal of Economics and Finance, 15(2), 455-472.

https://doi.org/10.21919/remef.v15i0.541

\section{Loaiza, Christian.}

2002

Mathews, John A.

Dragon Multinational: A New Model for Global Growth. Nueva York: Oxford University Press

\section{Mignolo, Walter D., \& Schiwy,} Freya.

Double Translation: Transculturation and the Colonial Difference. Translation and Ethnography: The Anthropological Challenge of Intercultural Understanding (pp. 6-28). Tucson: University of Arizona Press.

\section{Morales, Mario, Amaro,} Marcela, \& Stezano, Federico A.

Tendencias tecnológicas en el sector biotecnológico: análisis de patentes en México y Estados Unidos. Economía Teoría y Práctica, 51, 17-44.

\section{Moreno, Ángel, \& Espinosa,} Rafael S.

Effects of the Foreign Direct Investment on the Productivity of Latin American Countries (1990-2012). Economía Teoría y Práctica, 49, 7-36.

\section{Naciones Unidas.}

Nuestro Futuro Común. Comisión Mundial Sobre el Medio Ambiente y el Desarrollo. https://www.un.org/es/ga/president/65/ issues/sustdev.shtml

\section{Naciones Unidas.}

Objetivos de Desarrollo Sostenible. https:// www.undp.org/content/undp/es/home/ sustainable-development-goals.html

\section{Ndhlovu, Finex.}

A decolonial critique of diaspora identity theories and the notion of superdiversity. 


\section{bibliografía}

Diaspora Studies, 9(1), 28-40. https://doi.or g/10.1080/09739572.2015.1088612

\section{Orellana, Carlos, \& Rivera,} Maribel.

2019

Contexto Sociopolítico y Marco Institucional de los Saberes Ancestrales y el Trabajo Artesanal en Ecuador. Perspectivas, (13), 58-77

\section{Quijano, Aníbal.}

$2000 a$

Colonialidad del poder y clasificación social. Journal of World-System Research, 11(2), 342-386.

\section{Quijano, Aníbal.}

2000b Colonialidad del poder, eurocentrismo y América Latina. En Edgardo Lander (comp.), La colonialidad del saber: eurocentrismo y ciencias sociales. Perspectivas Latinoamericanas. Buenos Aires: Clacso, Consejo Latinoamericano de Ciencias Sociales. http://www. decolonialtranslation.com/espanol/quijanocolonialidad-del-poder.pdf

\section{Quijano, Aníbal.}

2000c Coloniality of Power and Eurocentrism in Latin America. International Sociology, 15(2), 215-232

\section{Quijano, Aníbal.}

2014 Cuestiones y horizontes: De la dependencia histórico estructural a la colonialidad/descolonialidad del poder. Buenos Aires: Clacso.

\section{Ramamurti, Ravi.}

What is really different about emerging market multinationals? Global Strategy Journal, 2, 41-47.

Saavedra-Mayorga, Juan, Gonzales-Miranda, Diego R., \& Marín-Idárraga, Diego A.

2017

Teoría Crítica y Postmodernismo en los Estudios Organizacionales en América Latina. En S. R. Clegg, C. Hardy, T. B. Lawrence, W. R. Nord, G. Ramírez Martínez y D. R. Gonzales-Miranda (eds.), Tratado de estudios organizacionales (vol. I, pp. 553-590). Medellín: UAM, Remineo, Editorial Universidad Eafit, SAGE.

\section{Smith, Andrea.}

Indigeneity, Settler Colinialism, White Supremacy. En Racial Formation in the Twenty-first Century (pp. 66-90). Berkeley: University of California Press.

\section{Tatsuo, Alejandro, \& Aguilera,} Maria Natividad.

Distancia Psicológica. Economía Teoría y Práctica, 46, 173-194.

\section{Taylor, P. J.}

Foreword: A debate on the Significance of 1492. En The Debate on Colonialism, Eurocentrism and History. Africa World Press

\section{Tuck, Eve, \& Yang, K. Wayne.}

2012 Decolonization is not a metaphor. Decolonization: Indigeneity, Education \& Society, 1(1), 1-40.

\section{Weick, Karl E.}

Drop your Tools: An Allegory for Organizational Studies. Administrative Science Quarterly, 41(2), 301-313.

\section{Wills, Charles W.}

2013 White Supremacy as Sociopolitical System: A Philosophical Perspective. White Out: 


\section{bibliografía}

The Continuing Significance of Racism

(pp. 35-48). Nueva York: Routledge.

Zhang, Yijing, Toppinen, Anne,

\section{\& Uusivuori, Jussi.}

2014 Internationalization of the forest product

industry: A synthesis of literature and

implications for future research. Forest

Policy and Economics, 38, 8-16.

Fecha de recepción: 12 de febrero de 2021

Fecha de aprobación: 25 de agosto de 2021

Fecha de publicación: 25 de octubre de 2021 